\title{
FORMAÇÃO DE PROFESSORES DA EDUCAÇÃO BÁSICA PARA A CAPTAÇÃO DE DOADORES DE SANGUE DO FUTURO
}

\section{TRAINING OF BASIC EDUCATION TEACHERS FOR THE CAPTURE OF BLOOD DONORS OF THE FUTURE}

\author{
José Benedito dos Santos Batista Neto ${ }^{1} *$ Thiago Marcírio Gonçalves de Castro $^{2} *$ Livia Caroline $^{2}$ \\ Machado da Silva ${ }^{*}$ Caio Heitor Vieira Melo ${ }^{*}$ Silvio Henrique dos Reis Júnior ${ }^{5} *$ Renata Campos de $^{*}$ \\ Sousa Borges $^{6} *$ Milena Coelho Fernandes Caldato $^{7}$
}

\begin{abstract}
RESUMO
Objetivo: relatar uma experiência sobre a realização de ações para a formação de professores para a captação de doadores do futuro em escolas públicas do município de Tucuruí, Pará. Método: Trata-se de um estudo descritivo, do tipo relato de experiência oriundo da realização de um projeto de extensão universitária interdisciplinar e intersetorial desenvolvido pela Universidade do Estado do Pará em parceria com o serviço de saúde de média complexidade Fundação Centro de Hemoterapia e Hematologia do Pará, o qual consistiu na realização de ações alusivas a formação de professores para a captação de doadores do futuro em escolas públicas da educação básica. Resultados: a experiência aconteceu em etapas: 1) revisão de literatura para aprofundamento acerca da problemática e aquisição de conhecimentos sobre soluções; 2) planejamento e organização de materiais e atividades e 3) execução das ações. As temáticas abordadas durante as formações foram: contexto histórico da doação sanguínea, conceituação e importância da transfusão sanguínea, dados estatísticos sobre doação, funcionamento de um hemocentro e etapas de uma doação, mitos e verdades sobre a doação. Considerações Finais: a experiência conseguiu despertar interesse nos professores pelo tema, portanto, conclui-se que os alunos também serão agraciados com o conhecimento, vindo a tornarem-se possíveis doadores.
\end{abstract}

Palavras-chave: Doadores de Sangue; Educação em Saúde; Docentes; Saúde Escolar; Promoção da Saúde.

\begin{abstract}
Objective: to report an experience on the performance of actions for teacher training to raise future donors in public schools in the city of Tucuruí, Pará. Method: This is a descriptive study, of the type of experience report derived from the realization of an interdisciplinary and intersectoral university extension project developed by the State University of Pará in partnership with the medium complexity health service Foundation of Hemotherapy and Hematology Center of Pará, which consisted of actions alusive to the training of teachers to capture donors of the future in public schools of basic education. Results: the experience took place in stages: 1) literature review to deepen the problem and acquisition of knowledge about solutions; 2) planning and organization of materials and activities and 3) execution of actions. The themes addressed during the training were: historical context of blood donation, conceptualization and importance of blood transfusion, statistical data on donation, functioning of a hemocenter and stages of a donation, myths and truths about donation. Final Considerations: the experience managed to arouse interest in teachers by the theme, therefore, it is concluded that students will also be awarded with knowledge, becoming possible donors.
\end{abstract}

Keywords: Blood Donors; Health Education; Faculty; School Health; Health Promotion.

\footnotetext{
${ }^{1}$ Universidade do Estado do Pará (UEPA), Tucuruí, Pará, Brasil. ORCID: https://orcid.org/0000-0003-3228-2340

${ }^{2}$ Universidade do Estado do Pará (UEPA), Tucuruí, Pará, Brasil. ORCID: https://orcid.org/0000-0001-8069-8899

${ }^{3}$ Universidade do Estado do Pará (UEPA), Tucuruí, Pará, Brasil. ORCID: https://orcid.org/0000-0002-7356-4005

${ }^{4}$ Universidade do Estado do Pará (UEPA), Tucuruí, Pará, Brasil. ORCID: https://orcid.org/0000-0001-5813-6195

${ }^{5}$ Universidade do Estado do Pará (UEPA), Tucuruí, Pará, Brasil. ORCID: https://orcid.org/0000-0002-5795-9453

${ }^{6}$ Universidade do Estado do Pará (UEPA), Tucuruí, Pará, Brasil. ORCID: https://orcid.org/0000-0002-7510-5582

${ }^{7}$ Universidade do Estado do Pará (UEPA), Belém, Pará, Brasil. ORCID: https://orcid.org/0000-0002-7077-8470
} 


\section{INTRODUÇÃO}

A doação de sangue é um ato voluntário, no qual o doador permite a retirada de parte de seu sangue, para armazenamento em bancos de sangue e/ou hemocentros, os quais, posteriormente, poderão utilizá-lo para transfusões ${ }^{(1)}$. Até os dias atuais, essa prática, é um problema de interesse mundial, pois não há uma substância que possa, em sua totalidade, substituir o tecido sanguíneo ${ }^{(2)}$. Assim sendo, a doação é a única possibilidade terapêutica para cura e reabilitação de inúmeros indivíduos que necessitam do processo de transfusão.

Nesse sentido, é fundamental que os hemocentros estejam sempre com seus estoques em níveis que possam atender a demanda da comunidade. Entretanto, esta não é a realidade de todos, haja vista que diversos países, inclusive o Brasil, enfrentam a problemática de possuir estoque abaixo da demanda ideal. Esta conjuntura explica-se por não assentirem com a comercialização sanguínea, sendo permitido apenas a doação voluntária, a qual, em muitos casos, não é bem difundida e/ou trabalhada ${ }^{(3)}$.

No Brasil, é preconizado pelo Ministério da Saúde que dentre a população total, $3 \%$ a $5 \%$ seja doadora de sangue, no entanto, dados nacionais apontam que esse quantitativo é de aproximadamente $2 \%$, mostrando-se insuficiente. Portanto, é necessário um maior esforço para a captação de doadores, com o intuito de reverter este quadro ${ }^{(4)}$.

Nesse contexto, pesquisas apontam que uma estratégia eficiente para a captação de doadores é a utilização do marketing social associado à educação em saúde. Logo, as ações de captação devem ser desenvolvidas de forma diferenciada, mobilizando grupos de referência ${ }^{(5)}$. Posto isso, trabalhar a captação de doadores durante a educação básica é crucial, dado que este é um ambiente com elevado público jovem, grupo ideal para se tornarem futuros doadores voluntários, considerando-se que, em sua maioria, são dinâmicos, idealistas, motivados e saudáveis, bem como possuem muitos anos de vida para doar ${ }^{(6)}$.

Para mais, a parceria intersetorial entre profissionais da saúde e professores da educação básica é imprescindível para a realização de ações de educação em saúde, tendo em vista que estes dois campos trabalhando juntos são fundamentais para a atenuação de problemáticas ${ }^{(7)}$, como a captação de doadores a longo prazo.

A partir disso, exemplificando a eficácia das estratégias que envolvem a parceria entre saúde e educação para a captação de doadores, destaca-se o Centro de Hematologia e Hemoterapia de Santa Catarina (HEMOSC), o qual passou a desenvolver inúmeros projetos de captação, como o "Projeto Escola/doador do futuro", no qual 
profissionais da saúde formam professores acerca de temas que circundam a doação de sangue, o que permite que estes incentivem seus alunos a tornarem-se doadores do futuro. Tal estratégia, comprovadamente, possibilita que o HEMOSC disponha de um bom quantitativo de doadores, em sua maioria, jovens ${ }^{(8)}$.

Diante disso, este trabalho possui como objetivo relatar uma experiência sobre a realização de ações para a formação de professores para a captação de doadores do futuro em escolas públicas do município de Tucuruí, interior do estado do Pará.

\section{MÉTODOS}

Trata-se de um estudo descritivo, do tipo relato de experiência oriundo da realização de um projeto de extensão universitária interdisciplinar e intersetorial desenvolvido pela Universidade do Estado do Pará (UEPA) em parceria com o serviço de saúde de média complexidade Fundação Centro de Hemoterapia e Hematologia do Pará (HEMOPA), o qual consistiu na realização de ações alusivas a formação de professores para a captação de doadores do futuro em escolas públicas da educação básica. Trabalhos do tipo relato de experiência são considerados uma ferramenta da pesquisa descritiva pautada em uma reflexão de ações que se referem a situações vivenciadas no âmbito profissional de interesse da comunidade científica ${ }^{(9)}$.
A experiência aconteceu em duas escolas públicas, Escola Municipal de Ensino Fundamental Manoel Carlos Silva e Escola Estadual de Ensino Médio Ana Pontes Francez, situadas nas zonas periféricas e central, respectivamente, do município de Tucuruí-PA. Para mais, a escolha pelas duas instituições adveio do fato de estarem localizadas distantes uma da outra, bem como por possuírem amplos espaços para a socialização das atividades, o que permitiu que um número considerável de professores pudesse participar.

Todo o processo, desde o planejamento até execução das ações, aconteceu entre os meses de agosto a outubro de 2019. A experiência foi executada por uma enfermeira e um biomédico (docentes e orientadores do projeto de extensão), quatro acadêmicos de Enfermagem, um acadêmico de Biologia e um acadêmico de Educação Física da UEPA. Ainda, contou com a parceria do HEMOPA, o qual disponibilizou duas assistentes sociais, que acompanharam todo o processo de realização das atividades.

Ademais, o público-alvo das ações foi de, aproximadamente, 40 professores das duas escolas públicas do município de Tucuruí-PA.

Foram respeitadas as resoluções 466/2012 e 510/2016 do Conselho Nacional de Saúde, assim sendo, por se tratar de um relato de experiência dos próprios autores não houve a necessidade de submissão do estudo à um comitê de ética em pesquisa, dado que não 
houve a manipulação de dados de seres humanos.

\section{RESULTADOS E DISCUSSÃO}

As ações de capacitação do projeto de extensão universitária, aqui relatadas, fazem parte do Programa Campus Avançado da UEPA, o qual incentiva a realização de ações extensionistas que contribuam para $o$ desenvolvimento local e atenuação de problemáticas da comunidade na qual a universidade está localizada. Desse modo, a ideia de realização do projeto surgiu após uma visita técnica dos docentes orientadores e dos acadêmicos ao HEMOPA de Tucuruí-PA, onde visualizaram o baixo número de bolsas de sangue disponíveis para transfusões. Com isso, a equipe de assistência social da instituição propôs a realização de uma parceria com a universidade, para $\mathrm{o}$ desenvolvimento do presente projeto de captação de doadores para atenuar a problemática.

Ademais, para melhor entendimento, a experiência aconteceu em três etapas: 1) realização de revisão de literatura para aprofundamento acerca da problemática e aquisição de conhecimentos sobre possíveis soluções; 2) planejamento e organização de materiais e atividades e 3) execução das ações. Ainda, os objetivos principais da experiência foram: difundir conhecimento sobre a importância da doação sanguínea e capacitar professores da rede pública de ensino de Tucuruí-PA para que se tornem captadores de doadores de sangue do futuro e assim, posteriormente, possibilitar que o HEMOPA aumente seu estoque de bolsas de sangue.

\section{Aprofundamento acerca da problemática e possível solução}

Com auxílio dos docentes orientadores, os acadêmicos realizaram pesquisas e leituras de artigos indexados em bancos de dados científicos, como Scientific Electronic Library Online (SciELO) e Medical Literature Analysis and Retrieval System Online (MEDLINE), para aquisição de aprofundamento teórico acerca da problemática, bem como de possíveis soluções.

Para a busca dos artigos científicos nos bancos de dados, foram utilizados os seguintes Descritores em Ciências da Saúde da Biblioteca Virtual em Saúde (DeCS/BVS): Doadores de Sangue/Blood Donor; Educação em

Saúde/Health

Education;

Docentes/Faculty; Educação Escolar/School Health. Ademais, para realização de combinações e associações entre esses descritores, tanto em língua portuguesa, quanto em língua inglesa, foi utilizado o operador booleano “AND”.

Assim sendo, foram lidos 18 artigos científicos nacionais e internacionais, que tratavam de temáticas que circundam a explanação de metodologias utilizadas para a 
captação de doadores de sangue, bem como a importância da parceria entre profissionais de saúde e educação no processo de realização de ações educativas que visem a promoção da saúde.

A partir do aprofundamento teórico e discussão entre os integrantes, chegou-se à conclusão da realização de ações de formação em saúde com professores da rede da educação básica do município, para que estes adquirissem conhecimentos sobre a doação e sua importância e assim se tornassem incentivadores de seus alunos, instigando-os a se tornarem doadores de sangue.

\section{Planejamento e organização de materiais e} atividades

Em um segundo momento, os acadêmicos juntaram-se a equipe de assistência social do HEMOPA para planejamento e organização dos materiais que seriam utilizados e das atividades que seriam realizadas durante a formação com os professores. A partir disso, foram construídas apresentações de slides e elaboradas dinâmicas para serem desenvolvidas. No mais, o HEMOPA disponibilizou folhetos, cartazes e cartilhas acerca da temática.

Com as atividades devidamente planejadas e organizadas, os acadêmicos iniciaram o processo de mobilização do público-alvo das ações. Portanto, em dias prédeterminados, deslocaram-se a todas as instituições de ensino do município, nas quais realizaram o convite aos docentes para que participassem da experiência.

Neste momento, também fora decidido que a formação com os professores aconteceria em duas escolas, em momentos distintos, para que assim um maior número de profissionais fosse contemplado com a experiência. Salienta-se que, fora realizada a mesma formação nas duas instituições.

\section{Execução das ações}

Durante a execução das ações houve a participação de, aproximadamente, 40 professores da rede pública de ensino e a carga horária total de cada encontro foi de 7 horas.

No que tange a realização da formação, inicialmente, os acadêmicos apresentaram-se e explanaram acerca dos objetivos da experiência, bem como pediram para que cada professor também se apresentasse, com o intuito de promover a interação entre os participantes. Em seguida, foram distribuídos os folhetos, as cartilhas e os cartazes disponibilizados pelo HEMOPA, nos quais continham informações referentes a formação. Sobre os cartazes, foi distribuído um quantitativo suficiente para cada profissional fixar nas paredes da escola em que trabalha.

Por conseguinte, era iniciada a formação mediada pelos slides e dinâmicas, a qual abrangeu cinco temáticas: contexto histórico da doação sanguínea, conceituação e 
importância da transfusão sanguínea, dados estatísticos sobre doação, funcionamento de um hemocentro, etapas de uma doação e mitos e verdades sobre a doação (Quadro 1).

Quadro 1 - Cronograma com cada temática abordada com os professores na formação para a captação de doadores de sangue do futuro, Pará, Brasil, 2021.

\begin{tabular}{|c|c|c|c|}
\hline Temáticas & Objetivos & $\begin{array}{l}\text { Métodos de } \\
\text { abordagem }\end{array}$ & Tempo \\
\hline $\begin{array}{l}\text { Apresentação } \\
\text { inicial/Abertura da } \\
\text { formação } \\
\text { distribuição de } \\
\text { materiais informativos }\end{array}$ & $\begin{array}{l}\text { Elencar os objetivos } \\
\text { da experiência }\end{array}$ & $\begin{array}{l}\text { a) Conversa dialogada } \\
\text { e b) apresentação } \\
\text { individual de cada } \\
\text { participante }\end{array}$ & $\begin{array}{lr}30 \quad \text { minutos. } \\
\text { Sendo } & 15 \\
\text { minutos } & \text { para } \\
\text { "a" e } & 15 \\
\text { minutos } & \text { para } \\
\text { "b" } & \end{array}$ \\
\hline $\begin{array}{l}\text { Palestra 1: Contexto } \\
\text { histórico da doação } \\
\text { sanguínea; }\end{array}$ & $\begin{array}{l}\text { Apresentar o percurso } \\
\text { histórico da doação a } \\
\text { nível internacional e } \\
\text { nacional }\end{array}$ & $\begin{array}{l}\text { c) Apresentação de } \\
\text { slides expositivos e d) } \\
\text { diálogo interativo com } \\
\text { os participantes }\end{array}$ & $\begin{array}{l}1 \text { hora e } 10 \\
\text { minutos. Sendo } \\
30 \text { minutos para } \\
\text { c) e } 40 \text { minutos } \\
\text { para d) }\end{array}$ \\
\hline $\begin{array}{l}\text { Palestra 2: } \\
\text { Conceituação } \\
\text { importância da } \\
\text { transfusão sanguínea }\end{array}$ & $\begin{array}{l}\text { Esclarecer do que se } \\
\text { trata a transfusão } \\
\text { sanguínea, } \\
\text { exemplificar quais } \\
\text { condições levam o } \\
\text { indivíduo a realizar } \\
\text { uma transfusão e } \\
\text { alertar sobre a sua } \\
\text { importância no } \\
\text { salvamento de vidas }\end{array}$ & $\begin{array}{l}\text { e) Apresentação de } \\
\text { slides expositivos e f) } \\
\text { diálogo interativo com } \\
\text { os participantes }\end{array}$ & $\begin{array}{l}1 \text { hora. Sendo } \\
30 \text { minutos para } \\
\text { e) e } 30 \text { minutos } \\
\text { para f) }\end{array}$ \\
\hline \multicolumn{4}{|c|}{ Intervalo de 20 minutos } \\
\hline $\begin{array}{lr}\text { Palestra 3: } & \text { Dados } \\
\text { estatísticos } & \text { sobre } \\
\text { doação } & \end{array}$ & $\begin{array}{l}\text { Evidenciar os baixos } \\
\text { índices de doadores } \\
\text { para o país e suas }\end{array}$ & $\begin{array}{l}\text { g) Apresentação de } \\
\text { slides expositivos e h) } \\
\text { diálogo interativo com }\end{array}$ & 50 minutos \\
\hline
\end{tabular}




\begin{tabular}{|c|c|c|c|}
\hline & implicações & os participantes & \\
\hline $\begin{array}{l}\text { Palestra } \\
\text { Funcionamento de um } \\
\text { hemocentro e etapas } \\
\text { de uma doação }\end{array}$ & $\begin{array}{l}\text { Descrever } \\
\text { funcionamento de um } \\
\text { hemocentro e as } \\
\text { etapas da doação }\end{array}$ & $\begin{array}{l}\text { i) Apresentação de } \\
\text { slides expositivos e j) } \\
\text { montagem de mapas } \\
\text { mentais }\end{array}$ & $\begin{array}{l}1 \text { hora. Sendo } \\
30 \text { minutos para } \\
\text { i) e } 30 \text { minutos } \\
\text { para j) }\end{array}$ \\
\hline 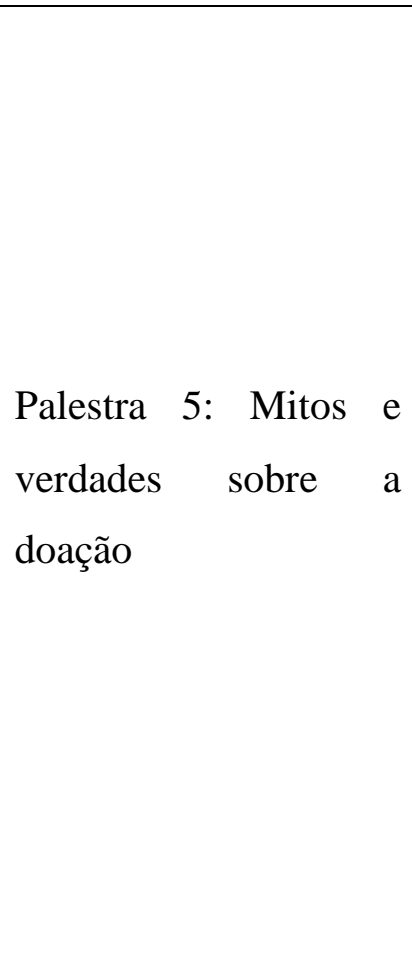 & $\begin{array}{l}\text { Esclarecer dúvidas } \\
\text { gerais acerca da } \\
\text { doação }\end{array}$ & $\begin{array}{l}\text { k) Dinâmica: cada } \\
\text { professor recebeu duas } \\
\text { placas, uma de "mito" } \\
\text { e outra de "verdade". } \\
\text { Após, foram lidas } \\
\text { informações que } \\
\text { deveriam ser julgadas } \\
\text { pelos docentes. } \\
\text { Roda de conversa para } \\
\text { esclarecimento de } \\
\text { possíveis na } \\
\text { cometidos erros } \\
\text { dinâmica }\end{array}$ & $\begin{array}{l}1 \text { hora e } 30 \\
\text { minutos }\end{array}$ \\
\hline $\begin{array}{l}\text { Encerramento com } \\
\text { feedback } \\
\text { experiência }\end{array}$ & $\begin{array}{l}\text { Encerrar a realização } \\
\text { da atividade e permitir } \\
\text { avaliação individual } \\
\text { dos participantes }\end{array}$ & $\begin{array}{l}\text { Roda de conversa para } \\
\text { feedback individual }\end{array}$ & 40 minutos \\
\hline
\end{tabular}

Fonte: Os autores.

Todas as temáticas foram abordadas por meio de métodos interativos e dinâmicos, que estimulavam os professores a participarem ativamente do processo, sempre dialogando entre si e fornecendo contribuições. É essencial que formações como esta relatada, prezem pela interação e diálogo, o que favorece o desenvolvimento crítico-reflexivo dos participantes, permitindo que estes tornem-se inteirados e articulados sobre determinado assunto ${ }^{(10)}$.

As três primeiras palestras foram mediadas pela apresentação expositiva de slides, associada ao diálogo interativo com os participantes sobre as temáticas abordadas. Salienta-se que tal metodologia fora adotada 
como forma eficiente de produzir conhecimento, dado que os indivíduos eram instigados a expor suas experiências e conhecimentos pessoais.

Sabe-se que, por anos, o processo educacional ficou aprisionado à métodos tradicionais, em que o conhecimento era repassado de forma passiva aos educandos, tornando-se massivo e pouco eficiente. Com o passar dos anos, as metodologias ativas ganharam espaço, estas, basicamente, estimulam a participação ativa dos alunos na construção de seu saber, para que isso ocorra, é necessário a criação de um ambiente colaborativo, no qual professores e alunos possuam lugar de fala, dialogando e trocando experiências entre si, favorecendo, então, a autorregulação e autonomia do aprendizado (11).

Ademais, a quarta palestra aconteceu por meio da apresentação expositiva de slide com associação de montagem de mapas mentais pelos participantes, como forma de facilitar o processo de ensino-aprendizagem do tema abordado. Mapas mentais são ferramentas, do tipo diagrama, construídas para que se alcance a gestão de informações/conhecimentos, onde é utilizada a organização visual de conceitos de forma criativa e simples. A eficiência dos mapas está associada ao fato de que para que sejam construídos, todas as habilidades e funções do cérebro do indivíduo são estimuladas, promovendo a elevação da produtividade e memorização ${ }^{(12)}$.

Por conseguinte, sobre a quinta palestra, tem-se que fora utilizado a dinâmica lúdica "Mitos e Verdades", em conjunto com a metodologia da roda de conversa. Acerca da dinâmica, sabe-se que é amplamente utilizada em experiências de educação em saúde, como observado na literatura científica existente $(13,14)$. O teor lúdico da atividade possibilita maior interação entre os indivíduos, favorece a troca de saberes e torna o processo de ensino-aprendizado mais significativo, além de proporcionar a quebra do senso comum e ratificação de informações equivocadas de forma descontraída ${ }^{(14)}$.

Ainda, tem-se que após a realização da dinâmica, fora realizada uma roda de conversa para esclarecimento dos erros cometidos. À priori, todos os indivíduos, sentados em roda, eram instigados a comentar acerca das respostas erradas, explicando a informação verdadeira. A roda de conversa é tida como um modelo informal de troca de conhecimentos, caracterizado como um espaço democrático, no qual todos os envolvidos colaboram para a edificação do processo educacional, por meio do diálogo e conversação. É uma forma de permitir que os alunos assumam uma posição ativa durante a construção do conhecimento ${ }^{(15)}$.

Após a finalização da formação, os docentes eram estimulados a avaliar a experiência, apontando os possíveis impactos 
que ela trouxera para suas vidas pessoal e profissional. Logo, cada participante deu um feedback da ação. O feedback é conceituado como uma ferramenta de avaliação formativa, amplamente utilizada após ações de educação em saúde, com o intuito de verificar, por meio das opiniões dos participantes, se o processo de ensino-aprendizagem foi eficiente e/ou alcançou um desempenho satisfatório. Ainda, a ferramenta visa verificar a necessidade de uma nova realização da ação e/ou garantir melhorias para ações futuras ${ }^{(16)}$.

Destarte, os docentes traçaram comentários positivos ao momento. Diversos afirmaram que englobariam a temática em suas aulas de ciências, sociologia, história, entre outras, além fazerem elogios à iniciativa, incentivando que experiências como estas fossem replicadas em outros momentos e escolas. Neste contexto, foi perceptível que os profissionais foram convencidos sobre a importância da temática e a necessidade do incentivo precoce para que os alunos da educação básica se tornem futuros doadores de sangue e que possam repassar essas informações a outros indivíduos, tornando-os multiplicadores de conhecimento. Assim, associar a prática educacional com temáticas que circundam a saúde é essencial para a solução de problemáticas e transformação da realidade (17).

Por conseguinte, subentendeu-se que a atividade desenvolvida foi exitosa, uma vez que o Ministério da Saúde evidencia a importância da realização de educação em saúde nas escolas de educação básica para a captação e fidelização de doadores de sangue, dado que instituições educacionais podem funcionar como peças-chave para a criação do hábito e da cultura de doação por parte dos alunos ${ }^{(18)}$.

Para mais, temos que atividades que trabalham com a parceria entre instituições de ensino e saúde para a captação de doadores de sangue e promoção da saúde alcançam ótimos resultados, fomentando a aquisição de valores, estimulando o exercício da cidadania e propiciando a construção de saberes ${ }^{(19)}$.

Ademais, outro resultado a ser elencado acerca da realização da formação, é que professores capacitados para a captação de doadores são capazes desenvolver atividades que diminuem os medos e anseios dos alunos acerca da temática, fazendo-os criar consciência da importância do ato de doar para a sociedade que os cerca ${ }^{(4)}$.

\section{Limitações da experiência}

Acerca das limitações, aponta-se que durante a mobilização dos professores não foi possível abranger a todos, pois, muitos não se encontravam nas escolas nos horários em que os acadêmicos foram realizar o convite. No mais, a experiência não abrangeu docentes residentes e atuantes na zona rural do município. Além disso, existiu a limitação das ações ocorrerem apenas em dois momentos e 
locais. Acredita-se que, se mais escolas sediassem a experiência, o número de participantes seria maior.

Ainda, considera-se que além da utilização do feedback como método avaliativo do impacto da experiência, poderia ter sido realizada uma pesquisa de opinião, baseada em questionários, com os participantes, a qual permitiria mensurar o grau de conhecimento científico que a atividade trouxe aos docentes.

\section{Contribuições para a prática}

A contribuição desta experiência está ligada ao conhecimento apresentado aos docentes, os quais, tornaram-se habilitados para abordar a temática dentro de sala de aula e na comunidade, o que, certamente, auxiliará no processo de captação de doadores para o HEMOPA do município. É fundamental que entendamos a importância da escola e do professor em trabalharem temas da saúde com seus alunos, indo além da abordagem de temáticas que circundam a orientação e prevenção de doenças. A educação atrelada à saúde deve informar que os jovens são importantes agentes sociais, haja vista que suas decisões individuais, como a de se tornarem doadores, são essenciais para a evolução e desenvolvimento da sociedade em que estão inseridos ${ }^{(20)}$.

No mais, tem-se que a experiência também trouxe contribuições significativas aos acadêmicos envolvidos, em especial, aos de enfermagem, que adentraram em um campo ainda pouco explorado pela profissão, a hemoterapia, mas, que com certeza será de grande valia para a vida profissional futura, uma vez que também se tornaram captadores. Cabe ao enfermeiro, que trabalha com hemoterapia, incentivar a comunidade, por meio de estratégias educativas, sobre a importância da doação de sangue ${ }^{(21)}$.

\section{CONSIDERAÇÕES FINAIS}

Frente a problemática dos baixos índices de doação de sangue pelo país, faz-se necessário que estratégias sejam traçadas para que esta problemática seja revertida. Logo, temos que a experiência aqui relata, é uma boa alternativa, pois, conseguiu despertar o interesse nos professores pelo tema, dado que foi verbalizado em seus comentários positivos. Portanto, conclui-se que os seus alunos também serão agraciados com o conhecimento, tornando-se assim, possíveis doadores de sangue.

\section{REFERÊNCIAS}

1. Malheiros GC, Oliveira AAST, Pinheiro CB, Monteiro KNO, Abreu AMOW. Fatores associados à motivação da doação sanguínea. Revista Científica da FCM [Internet]. 2014 [cited 2020 Dec 30]; 9(1): 08-12. Available from:

http://www.fmc.br/ojs/index.php/RCFMC/articl e/view/55/44.

2. Rodrigues RSM, Reibnitz KS. Estratégias de captação de doadores de sangue: uma revisão integrativa da literatura. Texto \& Contexto Enfermagem [Internet]. 2011 [cited 2020 Dec 30]; 20(2): 384-391. Available from: 
https://www.scielo.br/scielo.php?script=sci_artt ext\&pid=S0104-07072011000200022.

3. Locks MOH, Salum NC, Barros BS, Matos E, Anders JC, Schneider DG. Perfil dos doadores de sangue que apresentaram reações adversas à doação. Revista Brasileira de Enfermagem [Internet]. 2019 [cited 2020 Dec 30]; 72(1): 81-87. Available from: https://www.scielo.br/pdf/reben/v72n1/pt_003 4-7167-reben-72-01-0081.pdf.

4. Ministério da Saúde (BR), Gabinete do Ministro. Portaria $\mathrm{n}^{\circ} 158$, de 04 de fevereiro de 2016. Aprova o Regulamento Técnico de Procedimentos Hemoterápicos [Internet]. Brasília: Ministério da Saúde; 2016 [cited 2020 Dec 30]. Available from: http://bvsms.saude.gov.br/bvs/saudelegi s/gm/2016/prt0158_04_02_2016.html.

5. Pereira JR, Sousa CV, Matos EB, Rezende LBO, Bueno NX, Dias AM. Doar ou não doar, eis a questão: uma análise dos fatores críticos da doação de sangue. Ciência \& Saúde Coletiva [Internet]. 2016 [cited 2020 Dec 30]; 21(8): 2475-2484.

Available from: https://www.scielo.br/pdf/csc/v21n8/14138123-csc-21-08-2475.pdf.

6. Özgür S, Ürek H, Kösal K. Turkish university students' opinions towards blood donation. Univeral Journal of Education Research [Internet]. 2018 [cited 2020 Dec 30]; 6(5): 897-908. Available from: http://www.hrpub.org/download/20180430/U JER11-19511201.pdf.

7. Silva LGS, Costa JB, Furtado LGS, Tavares JB, Costa JLD. Primeiros socorros e prevenção de acidentes no ambiente escolar: intervenção em unidade de ensino. Enfermagem em Foco [Internet]. 2017 [cited 2020 Dec 30]; 8(3): 25-29. Available from:

http://revista.cofen.gov.br/index.php/enferma gem/article/view/893.

8. Rosa LM, Rodrigues RSM, Nitschke RG, Silva RDN, Ferreira JC, Baldissera JLC. Captação de doadores e doação de sangue: discursos históricos. Revista de Enfermagem UFPE On Line [Internet]. 2018 [cited 2020 Dec 30]; 12(10): 2766-2774. Available from: https://doi.org/10.31011/reaid-2021-v.95-n.36-art.1187 Rev Enferm Atual In Derme v. 95, n. 36, 2021 e-021146

https://periodicos.ufpe.br/revistas/revistaenfer magem/article/download/234866/30248.

9. Pereira MO, Reinado AMS, Villa EA, Gonçalves AM. Superando os desafios para oferecer formação de qualidade em enfermagem psiquiátrica. Revista Brasileira de Enfermagem [Internet]. 2020 [cited 2021 Out 10]; 73(1):e20180208. Available from: https://www.scielo.br/j/reben/a/SZWXtvz3Zd Rr7hQ3zwDfpry/?format=pdf\&lang=pt.

10. Macedo KDS, Acosta BS, Silva EB, Souza NS, Beck CLC, Silva KKD. Metodologias ativas de aprendizagem: caminhos possíveis para inovação no ensino em saúde. Escola Anna Nery [Internet]. 2018 [cited 2021 Jan 27]; 22(3): e20170435. Available from: https://www.scielo.br/pdf/ean/v22n3/pt_14148145-ean-22-03-e20170435.pdf.

11. Paiva MRF, Parente JRF, Brandão IR, Queiroz AHB. Metodologias ativas de ensinoaprendizagem: revisão integrativa. SANARE - Revistas de Políticas Públicas [Internet] 2016 [cited 2021 Out 10]; 15(2): 145-153. Available

from: https://sanare.emnuvens.com.br/sanare/article/ view/1049.

12. Nascimento ALGP, Silveira R, Jesus RCC, Simões JB. Mapas mentais como ferramenta de ensino/aprendizagem para universitários com TDAH/TODA no curso de letras da FAE centro universitário. Memorial TCC - Caderno de Graduação [Internet] 2019 [cited 2021 Out 10]; 5(1): 403-436. Available from:

https://cadernotcc.fae.edu/cadernotcc/article/v iew/279/161.

13. Costa ECS, Fontoura Es, Souza SLC, Saraiva APC. Mito ou verdade? Educação em saúde com gestantes sobre aleitamento materno exclusivo. Revista Eletrônica Acervo Enfermagem [Internet] 2020 [cited 2021 Out 10]; 6: e5375. Available from: https://acervomais.com.br/index.php/enferma gem/article/view/5375.

14. Morais JR, Novaes JF, Azevedo FM, Teixeira BA, Campos IX, Coelho MF. Dinâmica "mitos e verdades" como estratégia 
de educação alimentar e nutricional de escolares: um trabalho de extensão realizado pelo Programa de Educação para o Trabalho do curso de Nutrição da Universidade Federal de Viçosa (PET-NUT/UFV). Journal of Management and Primary Health Care [Internet] 2016 [cited 2021 Out 10]; 7(1): 1212. Available from: https://jmphc.com.br/jmphc/article/view/338.

15. Melo ES, Aragaki SS. Roda de conversa como estratégia para a gestão e educação permanente em saúde. Revista Portal Saúde e Sociedade [Internet] 2019 [cited 2021 Out 10]; 4(2): 1152-1159. Available from: https://www.seer.ufal.br/index.php/nuspfame d/article/view/7819/6376.

16. Dose EMC. A importância do feedback na educação à distância. Revista on line de Política e Gestão Educacional [Internet] 2017 [cited 2021 Out 10]; 21(3): 1565-1571. Available from: https://periodicos.fclar.unesp.br/rpge/article/vi ew/10973/7108.

17. Simonetti SH, Massa VC, França JID. Método educativo convencional e inovador para o aprendizado no usuário de anticoagulação oral. Enfermagem em Foco [Internet] 2017 [cited 2021 Jan 27]; 8(4): 0306. Available from: http://revista.cofen.gov.br/index.php/enferma gem/article/view/938.

18. Ministério da Saúde (BR), Secretaria de Atenção à Saúde, Departamento de Atenção Especializada e Temática. Manual de orientações para a promoção da doação voluntária de sangue. [Internet] 2015 [cited 2021 Feb 09]; Brasília: Ministério da Saúde. Available from: https://bvsms.saude.gov.br/bvs/publicacoes/m anual_orientacoes_promocao_doacao_volunta ria_sangue.pdf.

19. Lima HF, Costa KC, Jerke LC, Portela JMG, Cogo SB, Silva LMC, et al. Educação em saúde sobre doação de sangue: relato de uma experiência com crianças e adolescentes. Research, Society and Development [Internet]. 2020 [cited 2021 Feb 09]; 9(9): e780997941. Available from: https://rsdjournal.org/index.php/rsd/article/vie $\mathrm{w} / 7941 / 7000$.

20. Silva RPN, Lara S, Copetti J, Lanes KG, Soares MC. Concepções de professores sobre os processos de educação em saúde no contexto escolar. Contexto \& Educação [Internet]. 2017 [cited 2021 Jan 27]; 103: 146-164. Available from: https://www.revistas.unijui.edu.br/index.php/c ontextoeducacao/article/view/6563/5582.

21. Oliveira FF. Reflexão sobre o saber e o fazer dos profissionais de enfermagem frente ao processo de hemoterapia. Revista Multitexto [Internet]. 2016 [cited 2021 Jan 27]; 4(2): 48-54. Available from: http://www.ead.unimontes.br/multitexto/index $. \mathrm{php} / \mathrm{rmcead} / \mathrm{article} / \mathrm{view} / 185$.

Autor correspondente: José Benedito dos Santos Batista Neto.

Endereço: Travessa São Benedito, ${ }^{\circ}$ 77,

Bairro Santa Maria, CEP: 68400-00, Cametá, Pará.

E-mail: netto1443@gmail.com

Submissão: 2021-07-31

Aprovado: 2021-10-11 